Title

\title{
Dopaminergic signaling supports auditory social learning
}

$\begin{array}{ll}\text { Authors } & \text { Nihaad Paraouty*1, Catherine } \\ & { }^{1} \text { Center for Neural Science } \\ & { }^{2} \text { Department of Psychology } \\ & { }^{3} \text { Department of Biology } \\ & { }^{4} \text { Neuroscience Institute, NY } \\ & \text { New York University } \\ & \text { New York, NY 10003 } \\ \text { Contact } & \text { *Nihaad Paraouty, Ph.D. } \\ & \text { Center for Neural Science } \\ & \text { New York University } \\ & 4 \text { Washington Place } \\ & \text { New York, NY 10003 } \\ & \text { Email np64@nyu.edu } \\ & \text { Phone 212-998-3924 }\end{array}$

$\begin{array}{ll}\text { Pages } & 28 \\ \text { Figures } & 4 \\ \text { Supplementary Figures } & 3 \\ \text { Video } & 1\end{array}$

\section{Key Words}

social learning, reward, dopamine, auditory discrimination

\section{Abbreviation}

amplitude modulation (AM)

\section{Acknowledgements}

The work was supported by the Fyssen Foundation (NP) and R01DC011284 (DHS).

\section{Author Contributions}

NP and CR performed the experiments; NP analyzed the data; NP and DHS designed the experiments and wrote the paper.

\section{Competing Interests}

The authors whose names are listed immediately above declare no competing interests. The authors certify that they have no affiliations with or involvement in any organization or entity with any financial, or nonfinancial interest in the subject matter or materials discussed in this manuscript. 


\section{Abstract}

2 Explicit rewards are commonly used to reinforce a behavior, a form of learning that engages the

3 dopaminergic neuromodulatory system. In contrast, skill acquisition can display dramatic improvements

4 from a social learning experience, even though the observer receives no explicit reward. Here, we test

5 whether a dopaminergic signal contributes to social learning in naïve gerbils that are exposed to, and learn

6 from, a skilled demonstrator performing an auditory discrimination task. Following five exposure sessions,

7 naïve observer gerbils were allowed to practice the auditory task, and their performance was assessed across

8 days. We first tested the effect of an explicit food reward in the observer's compartment that was yoked to

9 the demonstrator's performance during exposure sessions. Naïve observer gerbils with the yoked reward

10 learned the discrimination task significantly faster, as compared to unrewarded observers. The effect of this

11 explicit reward was abolished by administration of a D1/D5 dopamine receptor antagonist during the

12 exposure sessions. Similarly, the D1/D5 antagonist reduced the rate of learning in unrewarded observers. To

13 test whether a dopaminergic signal was sufficient to enhance social learning, we administered a D1/D5

14 receptor agonist during the exposure sessions in which no reward was present, and found that the rate of

15 learning occurred significantly faster. Finally, a quantitative analysis of observer vocalizations and

16 movements during the exposure sessions suggest behavioral strategies that contribute to social learning.

17 Together, these results are consistent with a dopamine-dependent reward signal during social learning. 


\section{Introduction}

19 A broad range of behavioral paradigms demonstrate that explicit rewards, such as food or money, can reinforce behaviors and facilitate learning (Hull, 1943; Ferster, 1957; Tempel et al., 1983; Schultz, 2000;

O’Doherty, 2004; Nakatani et al., 2009; Gottlieb et al., 2014; Berridge, 2007; Robbins and Everitt, 2007;

Steinberg et al., 2013). These reinforcing rewards typically engage dopaminergic signaling which acts to modulate both motivation and memory formation (Wickens et al., 2003; Schultz, 2002; Lisman and Grace, 2005; Calabresi et al., 2007; Rossato et al., 2009; Bromberg-Martin et al., 2010; Flagel et al., 2011). correlated with reward presentation, as well as reward anticipation (Schultz et al., 1997; Schultz, 2013). dopamine receptor-dependent long-term potentiation (Schultz, 2006; Calabresi et al., 2007; Adamantidis et al., 2011). Reward anticipation can also enhance attention and, in turn, boost the encoding of incoming sensory signals (Maunsell et al., 2004; Seitz and Watanabe, 2005; Sasaki et al., 2010). Thus, explicit rewards have a positive impact on the learning and retention of sensory and motor skills (Roelfsema et al., 2010; Wickens et al., 2003; Abe et al., 2011; Orban et al., 2011; Shmuelof et al., 2012; Galea et al., 2015).

The role of dopaminergic signaling in explicit reward learning suggests that dopamine may also be engaged by implicit rewards, such as those triggered by learning in the absence of any external feedback (Ripolles et al., 2016; 2018), or certain intrinsic motivational states such as curiosity (Gruber et al., 2014). In principle, implicit reward signals share some of the neural mechanisms that attend the acquisition of an external reward, including dopamine release in the nucleus accumbens (Radhakishun et al., 1988; Hernandes and Hoebel, 1990; Martel and Fantino, 1993; Roitman et al., 2004). For example, fluctuations in dopamine levels occur in rat nucleus accumbens when naïve animals observe the delivery of an explicit reward to a conspecific (Kashtelyan et al., 2014). In these experiments, the naïve observer rat experienced an initial increase in dopamine levels, followed by a decrease in the absence of an explicit reward. Similarly, Ripolles et al. $(2014 ; 2016)$ showed that when subjects successfully learn the meaning of new words presented in verbal contexts, in the absence of any explicit reward or feedback, they also experienced an increase in 
emotion-related physiological measures and subjective pleasantness ratings. Such learning in the absence of external reward or feedback was found to be causally related to synaptic dopamine availability (Ripolles et al., 2018). When the subjects were provided with a dopamine precursor or a dopamine antagonist, both learning and pleasantness ratings were shifted as compared to a placebo group. The precursor group showed enhanced learning and pleasantness ratings, while the antagonist group showed a decrease in learning and pleasantness ratings.

Social interactions, themselves, can act as rewards (Panksepp and Lahvis, 2017; Dölen et al., 2013; Hung et al., 2017). Access to social stimuli or social isolation both recruit the dopamine reward system (Gunaydin et al., 2014; Matthews et al., 2016; Tamir and Hughes, 2018). In the current study, we asked whether dopamine signaling during exposure to a performing conspecific could contribute to social learning, defined here as the acquisition or facilitation of new skills by observation or exposure to a conspecific performing a well-defined behavior (Carcea and Froemke, 2019; Paraouty et al., 2020). We previously reported that naïve gerbils acquire a sound discrimination task significantly faster when exposed for five days to a demonstrator that was performing the task, as compared to three different control groups (Paraouty et al., 2020). To test the hypothesis that social learning engages a dopamine-dependent reward signal that facilitates the subsequent acquisition of an auditory task, we used pharmacological loss- and gain-of-function manipulations of D1/D5 dopamine receptors in naïve observer gerbils during exposure sessions. Furthermore, to assess the behavioral signals that might contribute to social learning, we monitored the vocalizations and movements of both observer and demonstrator during the exposure sessions. Together, the results suggest that dopaminergic signaling is both necessary and sufficient to facilitate socially-mediated task acquisition, and one important social cue is the demonstrators' vocalizations at trial initiation. 


\section{Presence of an explicit reward improves social learning}

Our first objective was to determine whether an explicit reward could facilitate social learning. As described previously (Paraouty et al., 2020), demonstrator gerbils were first trained by an experimenter to perform a Go-Nogo amplitude modulation (AM) rate discrimination task. Briefly, the demonstrators were placed on controlled food access and trained to initiate each trial by placing their nose in a nose port. The Go stimulus (12-Hz AM noise) indicated the presence of a food reward at the food tray, while the Nogo stimulus (4-Hz AM noise) signaled the absence of a food reward. A discrimination performance metric, d-prime (d') was calculated for each session as $d^{\prime}=z($ hit rate $)-z$ (false alarm rate). Once animals performed the task with a d'

$>1.5$, they qualified as a demonstrator (for more details, see Methods Section).

Our previous results demonstrated that observation alone led to social learning (Paraouty et al., 2020), and we first replicated that finding. A naïve observer gerbil was placed adjacent to a previously-trained and performing demonstrator gerbil for 5 consecutive exposure sessions (Figure 1A, left, also see Video 1). The demonstrator compartment was separated from the observer compartment by a transparent divider, and possessed a nose port and a food tray, thereby allowing the demonstrator gerbil to initiate and perform trials. The naïve observer gerbil had access to all sensory cues emanating from the demonstrator, and to the Go and Nogo sounds delivered $1 \mathrm{~m}$ above the test cage. During each exposure session, the naïve observer gerbil was exposed to a minimum of 80 Go trials and 20 Nogo trials performed by the demonstrator (Figure 1B, brown lines). Following the fifth exposure session, the demonstrator was removed as well as the divider, and the naïve observer gerbil was permitted to practice the task on its own (Figure 1A, right). The sensitivity metric, d', was computed for all sessions during which the naïve observer gerbil performed $>15$ Nogo trials. On average, the naïve observer gerbils required $5.6 \pm 0.35$ days (mean \pm standard error) to perform the task at a criterion d' of 1.5 (Figure 1C, black lines). No significant difference was found between the number of days taken to reach the criterion d' by the current observers and those tested in Paraouty et al. (2020, Figure 1, 
previous finding that social learning facilitates acquisition of a sound discrimination task (Paraouty et al., 2020) as compared to 3 control conditions that were examined in the original study: 1) naïve gerbils deprived of any prior exposure before performing the task, 2) naïve gerbils exposed to the test cage alone for 5 days prior to performing the task, and 3) naïve gerbils exposed to a non-performing demonstrator for 5 days prior criterion d' of 1.5 as compared to the observer groups (see Figure 2 in Paraouty et al., 2020).

To test whether an explicit food reward could further enhance social learning, we next placed a food dispenser in the observation chamber (Figure 1D, left), and provided naïve observers with a food reward that was yoked to the demonstrator's performance during the five exposure sessions. In other words, a food pellet was delivered to both the demonstrator and the naïve observer when the demonstrator responded accurately on Go trials. The naïve observers in this case were also exposed to a minimum of 80 Go trials and 20 Nogo trials in each pairing session (Figure 1E, brown lines). On average, the naïve observer gerbils with an explicit yoked reward required $3.3 \pm 0.21$ days to perform at criterion (Figure $1 \mathrm{~F}$, orange lines) which was significantly faster, as compared to observation alone (in grey; Wilcoxon rank sum test, $\mathrm{X}^{2}(1)=12.87$, $p=0.0003)$. Therefore, an explicit food reward during the exposure sessions facilitates social learning.

\section{Decreasing dopamine receptor signaling diminishes social learning}

111 Next, we asked whether an intrinsic dopaminergic signal during the exposure sessions contributed to social learning. Prior to each exposure session, the naïve observer gerbils were briefly anaesthetized with isoflurane and given an intra-peritoneal injection of a D1/D5 dopamine receptor antagonist, SCH-23390 (0.03 mg/kg, Figure 2A, left) or an injection of saline (Figure 2B, left). Animals were allowed to recover for 15-20 minutes before the exposure session began. Following the five days of exposure, the naïve observer animals were allowed to practice the task, and no further injections were delivered (Figure 2A-B, right). When D1/D5 receptor were blocked during the exposure sessions, naïve observer gerbils took significantly longer to reach the criterion performance, as compared to the saline-injected observer animals (Figure 2C; Wilcoxon rank sum test, $\left.\mathrm{X}^{2}(1)=13.81, p=0.0002\right)$. The saline-injected animals did not differ significantly from the uninjected 
animals (from Figure 1C; Holm-Bonferroni-corrected post-hoc comparisons, $p=0.7805$ ), suggesting that

neither saline nor the handling of the animal for the injection influenced the rate of task acquisition. During

the first practice sessions, both trial initiation and hit rates were significantly poorer for the antagonist-

post-hoc comparisons, $p<0.05$ ), suggesting that the five exposure sessions did not benefit those animals.

Thus, a decrease in tonic dopamine level during the exposure sessions led to a significant decrease in the subsequent rate of task acquisition.

We also tested whether a decrease in an intrinsic dopaminergic signal could be compensated for by the presence of an external food reward during the exposure sessions. Two additional groups of naïve observer gerbils were briefly anaesthetized prior to each exposure session, and given an intra-peritoneal injection of a D1/D5 dopamine receptor antagonist, SCH-23390 (0.03 mg/kg, Figure 2D, left) or an injection of saline (Figure 2E, left). After recovery, both groups of naïve animals were provided with the exposure sessions, during which they received a food reward that was yoked to the demonstrator's performance. Following the five days of exposure, the yoked reward observer animals were allowed to practice the task, and no further injections were delivered (Figure 2D-E, right). Naïve observer gerbils took significantly longer to reach the criterion performance when D1/D5 receptor were blocked, despite the presence of the yoked food reward during exposure sessions, as compared to the saline-injected control group (Figure 2F; Wilcoxon rank sum test, $\left.\mathrm{X}^{2}(1)=8.67, p=0.0032\right)$. In addition, the saline-injected animals did not differ significantly from the uninjected animals with the yoked reward (from Figure 1F; Holm-Bonferroni-corrected post-hoc comparisons, $p=0.8861$ ), suggesting that neither saline nor the handling of the animal for the injection influenced the rate of task acquisition. Trial initiation and hit rates were significantly impacted during the first practice sessions for the antagonist-injected animals despite the presence of the yoked reward during the exposure sessions (see Supplementary Figure 2, Holm-Bonferroni-corrected post-hoc comparisons, $p<0.05$ ). Thus, these results confirm that a decrease in intrinsic tonic dopamine level during the exposure sessions lead to a significant decrease in the subsequent rate of task acquisition despite the presence of yoked food reward. 
observers with the yoked food reward did not perform significantly better than the one without the yoked food reward (Holm-Bonferroni-corrected post-hoc comparisons, $p=0.0807)$. Together, these results suggest a crucial role of intrinsic dopamine signaling in social learning.

\section{Pharmacological increase in tonic dopamine level facilitates social learning rate}

In order to test whether a high intrinsic dopamine level was enough to facilitate social learning, naïve observer animals received a D1/D5 dopamine receptor agonist during the exposure sessions. Prior to each exposure session, naïve observer gerbils were briefly anaesthetized and given either an intra-peritoneal injection of a D1/D5 dopamine receptor agonist, SFK-38393 (5.0 mg/kg, Figure 3A, left) or an injection of saline (Figure 3B, left). Animals were allowed to recover for 15-20 minutes before the exposure session began. Following the five days of exposure, the animals were allowed to practice the task, and no further injections were delivered (Figure 3A-B, right). When D1/D5 receptors were activated during exposure sessions, naïve observer gerbils subsequently learned the task significantly faster, as compared to a group of saline-injected animals (Figure 3C; Wilcoxon rank sum test, $\mathrm{X}^{2}(1)=9.84, p=0.0017$ ). Thus, an increase in intrinsic dopamine signaling during the exposure sessions led to enhanced task acquisition during the subsequent practice sessions.

\section{D1/D5 antagonist during exposure changes the behavioral pattern of the observer animals}

Video footage from the five daily exposure sessions was recorded (see Video 1) and analyzed for a subset of observer animals $(n=5)$ in each of the seven experimental groups tested. To standardize the analyses, we only included the frames obtained during the first 20 minutes of each exposure session. Both the observer animal's position (Supplementary Figure 1A) and its head orientation (Supplementary Figure 1C) were computed using DeepLabCut (Mathis et al., 2018, see Methods). On average, no significant difference was found between observer animals with or without a yoked reward (Supplementary Figure 1B, left, Holm-Bonferronicorrected post-hoc comparisons, $p=0.7859$ ) in terms of distance travelled during each exposure session. observer animals which received either saline or a dopamine agonist (Supplementary Figure 1B, middle, 
$p=0.0192$ and $p=0.0012$ ). In contrast, observers which received the dopamine agonist did not differ

significantly from the saline observer animals $(p=0.3424)$. Yoked reward observers with the dopamine

Figure 1B, right, $p=0.015)$.

The head orientation of all observer groups were preferentially towards the observer's food tray, whether or not a food tray was present in the observer's compartment during the exposure sessions. However, in the presence of the food tray, the yoked reward observers oriented their head significantly more towards the food tray as compared to observers without a food tray (Supplementary Figure 1D, left, $p=0.0011$ ). Interestingly, observers with the dopamine antagonist which moved significantly less, also showed less preference to the food tray location as compared to observers with saline or with dopamine agonist (Supplementary Figure 1D, middle,,$p<0.0001$ for both). Similarly, the yoked reward observers which received the dopamine antagonist showed a significantly reduced preference to the food tray as compared to the saline controls (Supplementary

Figure 1D, right, $p=0.0019)$. Although the dopamine antagonist impacted both locomotion and head orientation, the yoked reward observers with dopamine antagonist consumed a similar number of food pellets during the exposure sessions as the yoked reward observers with saline or the yoked reward uninjected observers $(p=0.776 ; \mathrm{p}=0.053$ ). In addition, for all observers in the yoked reward condition (uninjected, salineinjected, and antagonist-injected observers), no correlation was found between the mean number of pellets consumed by the observers during the five exposure days and the subsequent number of practice days taken to reach the criterion d' $(\mathrm{r}=0.17 ; p=0.399)$. Together, these results confirm that the trend towards reduced food intake of the antagonist-injected observers as compared to the uninjected animals during the exposure sessions was not a limiting factor for the subsequent learning delay during the practice sessions.

\section{The timing of vocalizations during exposure matches closely the onset of the sound stimuli}


the first 20 minutes of each of the exposure session. Interestingly, both demonstrator and observer animals vocalized during the exposure sessions (Figure 4A). In general, male observers displayed significantly longer

observers in the unyoked reward condition, $p=0.0006$; observers in the yoked reward condition, $p=0.0011$ ).

For all exposure sessions analyzed, $60 \%$ of the demonstrators' vocalizations were initiated around the time of trial initiation (i.e., nose-poke) and presentation of the Go or Nogo sound stimulus (Figure 4D, dark grey bars). While a similar temporal relationship was displayed by the naïve observers (Figure 4E), these animals produced fewer vocalizations around the presentation of the Go or Nogo sound stimulus ( $22 \%$ of all calls). Furthermore, while a majority of those demonstrators' calls occurred prior to the nose poke and sound onset, the majority of the observers' calls occurred after the sound onset (Figures 4D and 4E, respectively).

To further assess whether the timing of the demonstrators' vocalizations influenced the observers' rate of social learning, we asked whether there was a relationship between the proportion of demonstrators' calls that occurred within $\pm 0.5 \mathrm{~s}$ of the sound onset during the exposure sessions, and the observers' subsequent rate of task acquisition during the practice sessions. In fact, there was a strong positive correlation (Figure 4F; Pearson's correlation coefficient, $\mathrm{r}=-0.677, p<0.001)$. In contrast, no correlation was found between the percent of demonstrator calls that occurred outside of the sound onset (i.e., $>0.5 \mathrm{~s}$ before or after the Go/Nogo stimulus) and the subsequent learning rate of the observers (Figure $4 \mathrm{G} ; \mathrm{r}=-0.197, p=0.258$ ). Overall, these results suggest that the timing of the demonstrators' vocalizations with the sound onset during the exposure sessions is a possible social cue for the observers to learn in this particular context.

\section{Non-task factors do not account for differences in learning rates}


female observers were used. No significant difference was found between male and female gerbils in terms of number of days to reach a criterion d' of 1.5 , when combined across groups (Kruskal-Wallis $\mathrm{H}$ test, $\mathrm{X}^{2}(6)=$ $3.37, p=0.770)$ or within each group $(p>0.05)$. The mean postnatal age of the different observer groups did not differ significantly (Kruskal-Wallis $\mathrm{H}$ test, $\left.\mathrm{X}^{2}(6)=6.51, p=0.369\right)$. No significant group difference was found in terms of total number of trials performed and d' measures of the different demonstrator groups during the 5 exposure sessions (Kruskal-Wallis $\mathrm{H}$ test, $\mathrm{X}^{2}(6)=4.84, p=0.564 ; \mathrm{X}^{2}(6)=6.1, p=0.423$, respectively). Together, these results suggest that the differences in social learning rates could not be explained by non-task factors.

\section{Discussion}

Dopamine signaling plays a broad role in learning that results in acquisition of explicit rewards (review, Schultz, 2016), suggesting that it may play a general role in social forms of learning that do not yield an immediate reward. To test this idea, dopamine signaling was manipulated only during the periods of social exposure. We first established that an explicit food reward during social exposure could facilitate the subsequent rate of learning (Figure 1). We then tested whether a reduction in tonic dopamine signaling caused a reduction in social learning. We found that the rate of learning was significantly delayed when animals were treated with a D1 receptor antagonist during social exposure (Figure 2). Finally, to test whether tonic dopamine signaling was sufficient to facilitate social learning, in the absence of any external reward, animals were treated with a D1 receptor agonist during social exposure (Figure 3). This lead to faster task learning, suggesting a role for dopaminergic signaling during social learning itself, which is distinct from any dopaminergic signaling that may occur while animals practice the task and begin to receive an explicit food reward. Overall, our findings suggest that theories of dopamine-dependent learning can account for task acquisition paradigms in which explicit rewards, and reward prediction errors, are unavailable.

Our results are broadly consistent with the role of dopaminergic signaling during social interactions. Indeed, dopamine has been found to play a vital role in social transmission of food preferences (Matta et al., 2017; Rodriguiz et al., 2004). For example, social learning of food preference is impaired when observer mice 
receive a D1-type receptor antagonist, whereas a D2 receptor antagonist is ineffective (Choleris et al., 2011).

Similarly, dopaminergic signaling is involved in learned aggression in rats (Suzuki and Lucas, 2015).

Although the present study was not designed to assess the role of D1/D5 signaling on attention, it is plausible

have decreased the general arousal and attention of the observers to both the social and non-social cues (to the demonstrator animal and the sound stimuli, respectively), or led to an aversive response to the test cage (Acquas and Di Chiara, 1994) in addition to the decrease in internal dopamine signaling.

In our experiments, tonic dopamine levels in the naïve observers were modulated during each exposure session through systemic drug injections. However, dopamine signaling is often temporally precise at the moment when animals receive a reward or perceive a cue that predicts a reward (Schultz, 2000). Therefore, it is possible that our manipulations also enhanced or depressed the transient dopamine signals occurring during the exposure sessions. For example, increased firing of dopamine neurons in the observers could have been elicited by specific actions of the demonstrator, such as nose poking, or food acquisition, or vocalization. The yoked-reward observers in our study generally did not seek, or consume, the food pellet immediately after its delivery during the exposure sessions. Therefore, it is plausible that both a general increase in dopaminergic activity, as well as a temporally precise signal (demonstrators' vocalizations) could have elicited dopaminergic activity transients, and each served to facilitate an expectation for sound cues and potential rewards. Future work would require that dopamine neuron activity is monitored during the observation period to directly determine whether signaling is tonic or phasic.

Auditory forms of social learning have been particularly well-studied in songbirds (Mooney, 2009; Chen et al., 2016; Mennill et al., 2018; Narula et al., 2018 ; Yanagihara \& Yazaki-Sugiyama, 2019), where dopaminergic signaling is involved in both song learning and song production (Kubikova et al., 2009; Gale and Perkel, 2010). 
facilitate learning (Sasaki et al., 2006). Similarly, local stimulation of D1/D5 receptors in the auditory cortex has been found to boost auditory stimulus detection (Bao et al., 2001; Happel et al., 2014; Deliano et al., 2020).

As shown in Paraouty et al (2020), gerbils are able to use either visual or auditory cues during the exposure session, and learning would accrue from each. One intriguing possibility is that observers attend to the demonstrators' vocalizations which were closely linked to a behavior (nose poke) and the sound stimulus onset (Figure 4). We speculate that the timing of the demonstrators' calls could boost the observers' attention to both the nose-poke behavior and the sound stimuli, thereby promoting the formation of visual memories of the demonstrator's position near the poke, or auditory memories of a sound cue associated with a vocalization, or both. While demonstrators' vocalizations may convey crucial social cues to the observers, when matched with the onset of the auditory Go or Nogo stimuli, future experiments could assess the individual role of each social cue.

In the present study, the task to be learned through social exposure involved several discrete behaviors: trial initiation by nose poking, reward-seeking following a Go stimulus, and the withholding of reward-seeking following a Nogo stimulus. The movements of the naïve observer gerbils during the exposure sessions indicate some nascent forms of imitation, with a preferred head direction towards the food tray location (Supplementary Figure 1C-D), suggesting one element of the task that may be learned early on, even in the absence of visual cues (Ellard and Eller, 2009). This head orientation preference may be explained, at least in part, with (1) the cage design that was less wide than long, (2) the lateral position of rodent eyes which permit attention while at a right angle to visual objects, and (3) the gerbil's ability to attend to auditory cues 
308 The current results do not exclude the participation of other parallel neuromodulatory circuits. When learning

309 from conspecifics, the observer's attentional and memory resources are focused on the demonstrator's

310 exploration and performance of skilled behaviors (Hoppit and Laland, 2013). Therefore, it is likely that the

311 cholinergic and noradrenergic systems play prominent roles in the modulation of attention, arousal, memory

312 formation (Hasselmo, 1999; Fitzpatrick et al., 2019). Moreover, social interactions during the exposure

313 sessions likely engage oxytocin and serotonin signaling (Dölen et al., 2013). Ultimately, it will be necessary

314 to assess the relative impact of each mechanism on the neural plasticity mechanisms that support social

315 learning. 


\section{Methods}

\section{Experimental animals}

318 Gerbil (Meriones unguiculatus, $\mathrm{n}=117$ ) pups were weaned at postnatal day $(\mathrm{P}) 30$ from commercial breeding

319 pairs (Charles River). Littermates were caged together, but separated by sex, and maintained in a $12 \mathrm{~h}$

320 light/dark cycle. All procedures related to the maintenance and use of animals were approved by the

321 University Animal Welfare Committee at New York University, and all experiments were performed in accordance with the relevant guidelines and regulations.

323

324

325

326

327

328

329

330

\section{Behavioral setup}

The behavioral setup was similar to Paraouty et al. (2020). Gerbils were placed in a plastic test cage (dimensions: $0.4 \times 0.4 \times 0.4 \mathrm{~m}$ ) that was housed in a sound attenuation booth (Industrial Acoustics; internal dimensions: $2.2 \times 2 \times 2 \mathrm{~m}$ ), and observed via a closed-circuit monitor. Auditory stimuli were delivered from a calibrated free-field tweeter (DX25TG0504; Vifa) positioned $1 \mathrm{~m}$ above the test cage. Sound calibration measurements were made with a $1 / 4$ inch free-field condenser recording microphone (Bruel \& Kjaer). A pellet dispenser (Med Associates Inc, $20 \mathrm{mg}$ ) was connected to a food tray placed within the test cage, and a nose port was placed on the opposite side. The nose port and food tray were equipped with IR emitters and sensors (Digi-Key Electronics; Emitter: 940 nm, 1.2V, 50 mA; Sensor: Photodiode 935 nm 5 nS). Stimuli, food reward delivery, and behavioral data acquisition were controlled by a personal computer through custom MATLAB scripts and an RZ6 multifunction processor (Tucker-Davis Technologies).

\section{Stimuli}

For the sound discrimination task, the Go stimulus consisted of amplitude modulated (AM) frozen broadband noise tokens ( $25 \mathrm{~dB}$ roll-off at $3.5 \mathrm{kHz}$ and $20 \mathrm{kHz}$ ) with a modulation rate of $12 \mathrm{~Hz}$ and a modulation depth of $100 \%$. The Nogo stimulus was similar to the Go stimulus, except for the modulation rate which was $4 \mathrm{~Hz}$. Both Go and Nogo stimuli had a 200 ms onset ramp, followed by an unmodulated period of 200 ms which then transitioned to an AM stimuli. The sound level used was $55 \mathrm{~dB}$ SPL. 


\section{Video recordings}

344 Videos of the test cage were captured with a Logitech c270-HD webcam (30 frames per second, Best Buy).

345 We used the open source software: DeepLabCut (Mathis et al., 2018) on a Windows 10 machine (Dell

346 Precision 5820, 64-bit operating system) to track the gerbil's position in the test cage during practice

347 sessions. The network was trained with 1,030,000 iterations using a total of 1064 labeled frames (labeling of

348 nose, left ear, right ear, and tail base) and tested on a set of 200 frames. Manual evaluation of labeling

349 accuracy was achieved by comparing the labels acquired from the network on the test set with the manual

350 labels.

\section{Sound recordings}

Audio recordings were captured with two Dodotronic microphones (Ultramic 384K_BLE), placed on either side of the test cage. We used the open source software: DeepSqueak (Coffey et al., 2019) on a Windows 10 machine (Dell Precision 5820, 64-bit operating system) to track the vocalizations of both the demonstrator animal and the observer animal in each session. DeepSqueak-screener was used to first create a library of gerbil calls. A detection network was then produced using this library of gerbil vocalizations. All audio recordings were analyzed using this custom gerbil detection network, followed by a post-hoc denoising stage and manual confirmation of all individual calls. Due to the physical barrier (i.e., the divider) separating the observer and demonstrator animal, only a subset of calls were caught by both speakers. Thus, the majority of calls ( $85 \%$ on average across all sessions) of the demonstrator was only caught by the demonstrator's microphone and the majority of calls ( $83 \%$ on average across all sessions) of the observer animal was only 


\section{Experimenter trained demonstrator gerbils}

367

Demonstrator gerbils $(n=43)$ were trained by the experimenters on a sound discrimination task. The demonstrators were placed on controlled food access prior to the start of training, and all animals were trained using an appetitive reinforcement operant conditioning procedure (see Paraouty et al., 2020). Animals first learned to approach the food tray and receive food pellets (Bio Serv) when the Go stimulus (12 Hz AM noise) was played. Animals were then trained to reliably initiate Go trials independently by placing their nose in the port. Once animals were performing a minimum of 80 Go trials with a hit rate $>80 \%$, Nogo trials were introduced. The probability of Nogo trials was kept at 30\% in order to keep the animal motivated to perform the task. Nogo trials were paired with a 4-second time-out during which the house lights were extinguished and the animal could not initiate a new trial. The presentation of Go and Nogo trials were randomized to avoid animals developing a predictive strategy. For more details on the experimenter training procedure, refer to Paraouty et al. (2020).

Responses were scored as a hit when animals approached the food tray to obtain a food reward upon Go trials. If animals re-poked or did not respond during the 5-second time window following a Go stimulus, it was scored a miss. During Nogo trials, responses were scored as a false alarm when animals incorrectly approached the food tray. If animals re-poked or did not respond during the 5-second time window following a Nogo stimulus, then it was scored a correct reject. Hit and FA rates were constrained to floor (0.05) and ceiling (0.95) values to avoid d' values that approach infinity. A performance metric, $d$ prime (d') was then calculated for each session by performing a z-transform of both Hit rate and False Alarm values: d' $=\mathrm{z}(\mathrm{Hit}$ rate) $-z$ (False Alarm rate) (Green and Swets, 1966). To qualify as a demonstrator, animals were required to perform the task with a d'> $>1.5$.

\section{Social learning paradigms}

During each exposure session (see Video 1), a demonstrator gerbil performed the discrimination task in the presence of a naïve, untrained observer gerbil $(n=74$, age $=96.9 \pm 32.7)$. No animals were excluded from the 
study. As shown in Paraouty et al., 2020 (Supplementary Figure 3), we found no significant difference between observers that were paired with either same-sex cagemates or same-sex non-cagemates. Thus, in the current study, we used both cagemates and non-cagemates demonstrators. In addition, the age difference between observer and demonstrator was identical for cagemates, or slightly older demonstrators for noncagemates. As in Paraouty et al., 2020 (Supplementary Figure 5), we did not observe a significant correlation between the demonstrator's performance nor age and the observer's subsequent performance on the task.

Both the demonstrator and the naïve observer gerbil were placed on controlled food access. A divider (acrylic sheet) was placed within the test cage to separate the demonstrator compartment from the observation or exposure compartment (see Paraouty et al., 2020). The naïve observer animal could thus see the demonstrator perform the task and was also exposed to the Go and Nogo sound stimuli as the speaker was located $1 \mathrm{~m}$ above the test cage. For the observers, a nose port and food tray were present only on the demonstrator's compartment, allowing the demonstrator to initiate and perform trials (Figure 1A, left). For the yoked reward observers, a food tray was also present in the observer's compartment, and the food reward of the demonstrator animal was yoked to that of the observer (Figure 1D, left). In both conditions, the naïve observers were exposed to a minimum of 80 Go trials and 20 Nogo trials performed by the demonstrator gerbils in each exposure session.

After five daily exposure sessions, the divider was removed after the final day of exposure, and the naïve observer gerbil was then allowed to practice the task (practice session). The observer's food tray (when present, i.e., for the yoked reward conditions) was also removed during the practice sessions. During the first and second practice sessions, the observer gerbil was given the benefit of no more than five experimentertriggered Go trials. These experimenter-triggered Go trials were initiated only when an animal was touching the nose port. This method of manually initiating Go trials was identical to the one used to train demonstrators, in order to maximize the animal's interest in the nose port object. Except for these experimenter-triggered Go trials, all Go trials were initiated by the gerbil. In order to limit a source of high 
performed $>25$ Hits. False alarm trials were paired with a 2-second time-out on the second day of Nogo trial introduction. For all following practice days, a 4-second time-out was used when animals false alarmed. A d' was computed for all practice sessions during which a minimum number of 15 Nogo trials were presented (see Paraouty et al., 2020). This was the standard procedure used for all observer groups tested here.

\section{Drug and saline injections}

Prior to each exposure session, a subset of naïve observer gerbils was briefly anaesthetized with isoflurane and given an intra-peritoneal injection of either physiological saline $(0.9 \% \mathrm{NaCl}, \mathrm{n}=13)$ or a $\mathrm{D} 1 / \mathrm{D} 5$ dopamine receptor antagonist, $\mathrm{SCH}-23390(0.025 \mathrm{mg} / \mathrm{kg}$, volume $=0.06 \mathrm{ml}, \mathrm{n}=10)$ or a $\mathrm{D} 1 / \mathrm{D} 5$ dopamine receptor agonist, SFK-38393 $(5.0 \mathrm{mg} / \mathrm{kg}$, volume=0.1 ml, $\mathrm{n}=11)$. In addition, a subset of yoked reward observer gerbils was injected with physiological saline $(0.9 \% \mathrm{NaCl}, \mathrm{n}=6)$ or the $\mathrm{D} 1 / \mathrm{D} 5$ dopamine receptor antagonist, $\mathrm{SCH}-23390(0.025 \mathrm{mg} / \mathrm{kg}$, volume $=0.06 \mathrm{ml}, \mathrm{n}=6)$. The doses were initially based from previous studies in gerbils (Barnes et al., 2016 ; Schicknick et al., 2008; 2012). Following injections, animals were allowed to fully recover in a recovery cage (for 15-25 minutes) before the exposure session began. Higher doses of both the agonist and antagonist produced noted behavioral and motor effects (excessive grooming and hyperactivity for the agonist, and reduced motor behavior for the antagonist).

\section{Performance measures and statistical analyses.}

Due to limited litter sizes, a given litter could not be split into all conditions. However, animals from one given litter was used for at least 2 conditions. In addition, for all groups, data was collected from at least 3 different litters in order to avoid any litter-specific biases. No litter differences were observed during the experimenter-training stages of the demonstrator animals. A performance measure (d') was calculated for each animal: $d^{\prime}=z($ Hit rate $)-z($ False Alarm rate $)$. Hit and False Alarm rates were constrained to floor $(0.05)$ and ceiling (0.95) values. To avoid high variance, $d$ ' was only computed for sessions in which the observer gerbil performed $>15$ Nogo trials. For the computation of the mean d' line, we used all values of d' and 
445 value could be computed). However, for all statistical tests and for the computation of the mean number of

446 days taken by each experimental and control group to reach a criterion d' of 1.5 , only actual d' values were

447 used. We first checked whether the number of days for each group to reach a criterion performance d' of 1.5

448 were normally distributed using the Shapiro Wilk test of normality. As the latter were not sufficiently

449 Gaussian, we chose to perform non-parametric tests. All group level statistical tests and effect size

450 calculations were performed using JMP Pro 14.0 on a Mac platform. Comparisons were carried out using the

451 Wilcoxon rank sum test or the Steel Dwass comparisons, as indicated. For comparisons of all groups tested,

452 one-way ANOVAs were computed followed by post-hoc multiple comparisons analyses, and alpha values

453 were Holm-Bonferroni-corrected. 


\section{References}

Abe, M., Schambra, H., Wassermann, E. M., Luckenbaugh, D., Schweighofer, N., \& Cohen, L. G. (2011). Reward improves long-term retention of a motor memory through induction of offline memory gains. Current Biology, 21(7), 557-562.

Acquas, E., \& Di Chiara, G. (1994). D1 receptor blockade stereospecifically impairs the acquisition of drugconditioned place preference and place aversion. Behavioural pharmacology.

Adamantidis, A. R., Tsai, H. C., Boutrel, B., Zhang, F., Stuber, G. D., Budygin, E. A., ... \& de Lecea, L. (2011). Optogenetic interrogation of dopaminergic modulation of the multiple phases of reward-seeking behavior. Journal of Neuroscience, 31(30), 10829-10835.

Bao, S., Chan, V. T., \& Merzenich, M. M. (2001). Cortical remodelling induced by activity of ventral tegmental dopamine neurons. Nature, 412(6842), 79-83.

Bellgrove, M. A., \& Mattingley, J. B. (2008). Molecular genetics of attention. In D. W. Pfaff \& B. L. Kieffer (Eds.), Annals of the New York Academy of Sciences: Vol. 1129. Molecular and biophysical mechanisms of arousal, alertness, and attention (p. 200-212). Blackwell Publishing.

Berridge, K. C. (2007). The debate over dopamine's role in reward: the case for incentive salience. Psychopharmacology, 191(3), 391-431.

Bromberg-Martin, E. S., Matsumoto, M., \& Hikosaka, O. (2010). Dopamine in motivational control: rewarding, aversive, and alerting. Neuron, 68(5), 815-834.

Calabresi, P., Picconi, B., Tozzi, A., \& Di Filippo, M. (2007). Dopamine-mediated regulation of corticostriatal synaptic plasticity. Trends in neurosciences, 30(5), 211-219.

Carcea, I., \& Froemke, R. C. (2019). Biological mechanisms for observational learning. Current opinion in neurobiology, 54, 178-185.

Chen, Y., Matheson, L. E., \& Sakata, J. T. (2016). Mechanisms underlying the social enhancement of vocal learning in songbirds. Proceedings of the National Academy of Sciences, 113(24), 6641-6646.

Choleris, E., Clipperton-Allen, A.E., Gray, D.G., Diaz-Gonzalez, S., Welsman, R.G. (2011). Differential effects of dopamine receptor D1-type and D2-type antagonists and phase of the estrous cycle on social learning of food preferences, feeding, and social interactions in mice. Neuropsychopharmacology 36: 16891702 .

Coffey, K. R., Marx, R. G., \& Neumaier, J. F. (2019). DeepSqueak: a deep learning-based system for detection and analysis of ultrasonic vocalizations. Neuropsychopharmacology, 44(5), 859-868.

Deliano, M., Brunk, M. G., El-Tabbal, M., Zempeltzi, M. M., Happel, M. F., \& Ohl, F. W. (2020). Dopaminergic neuromodulation of high gamma stimulus phase-locking in gerbil primary auditory cortex mediated by D1/D5-receptors. European Journal of Neuroscience, 51(5), 1315-1327.

Dölen, G., Darvishzadeh, A., Huang, K. W., \& Malenka, R. C. (2013). Social reward requires coordinated activity of nucleus accumbens oxytocin and serotonin. Nature, 501(7466), 179-184.

Ellard, C. G. \& Eller, M. C. Spatial cognition in the gerbil: Computing optimal escape routes from visual threats. Anim. Cogn. 12, 333-345 (2009).

Ferster, C. B. (1957). Concurrent schedules of reinforcement in the chimpanzee. Science.

Fitzpatrick, C. M., Runegaard, A. H., Christiansen, S. H., Hansen, N. W., Jørgensen, S. H., McGirr, J. C., ... \& Gether, U. (2019). Differential effects of chemogenetic inhibition of dopamine and norepinephrine neurons in the mouse 5-choice serial reaction time task. Progress in Neuro-psychopharmacology and Biological Psychiatry, 90, 264-276.

Flagel, S. B., Clark, J. J., Robinson, T. E., Mayo, L., Czuj, A., Willuhn, I., ... \& Akil, H. (2011). A selective role for dopamine in stimulus-reward learning. Nature, 469(7328), 53-57. 
498 Gale, S. D., \& Perkel, D. J. (2010). A basal ganglia pathway drives selective auditory responses in songbird dopaminergic neurons via disinhibition. Journal of Neuroscience, 30(3), 1027-1037.

Galea, J.M., Mallia, E., Rothwell, J., \& Diedrichsen, J. (2015). The dissociable effects of punishment and reward on motor learning. Nature neuroscience, 18(4), 597.

Gottlieb, J., Hayhoe, M., Hikosaka, O., \& Rangel, A. (2014). Attention, reward, and information seeking. Journal of Neuroscience, 34(46), 15497-15504.

Green, D. M., \& Swets, J. A. (1966). Signal detection theory and psychophysics (Vol. 1). New York: Wiley.

Gruber, M. J., Gelman, B. D., \& Ranganath, C. (2014). States of curiosity modulate hippocampus-dependent learning via the dopaminergic circuit. Neuron, 84(2), 486-496.

508 (2014). Natural neural projection dynamics underlying social behavior. Cell, 157(7), 1535-1551.

Happel, M. F., Deliano, M., Handschuh, J., \& Ohl, F. W. (2014). Dopamine-modulated recurrent corticoefferent feedback in primary sensory cortex promotes detection of behaviorally relevant stimuli. Journal of neuroscience, 34(4), 1234-1247.

Hasselmo, M. E. (1999). Neuromodulation: acetylcholine and memory consolidation. Trends in cognitive 513 sciences, 3(9), 351-359.

514 Hernandez, L., \& Hoebel, B. G. (1990). Feeding can enhance dopamine turnover in the prefrontal

515 cortex. Brain research bulletin, 25(6), 975-979.

516 Hoppitt, W., \& Laland, K. N. (2013). Social learning: an introduction to mechanisms, methods, and models.

517 Princeton University Press.

518 Hull, C. L. (1943). Principles of behavior (Vol. 422). New York: Appleton-century-crofts.

Hung, L.W., Neuner, S., Polepalli, J.S., Beier, K.T., Wright, M., Walsh, J.J., .. \& Malenka, R.C. (2017). 2564-2568.

Lisman, J. E., \& Grace, A. A. (2005). The hippocampal-VTA loop: controlling the entry of information into long-term memory. Neuron, 46(5), 703-713.

Martel, P., \& Fantino, M. (1996). Mesolimbic dopaminergic system activity as a function of food reward: a microdialysis study. Pharmacology Biochemistry and Behavior, 53(1), 221-226.

Mathis, A., Mamidanna, P., Cury, K. M., Abe, T., Murthy, V. N., Mathis, M. W., \& Bethge, M. (2018).

DeepLabCut: markerless pose estimation of user-defined body parts with deep learning. Nature neuroscience, 21(9), 1281-1289.

Matta, R., Tiessen, A. N., \& Choleris, E. (2017). The role of dorsal hippocampal dopamine D1-type receptors in social learning, social interactions, and food intake in male and female mice. Neuropsychopharmacology, 42(12), 2344-2353. (4), 617-631.

539 Maunsell, J. H. (2004). Neuronal representations of cognitive state: reward or attention?. Trends in cognitive 
Mennill, D. J., Doucet, S. M., Newman, A. E., Williams, H., Moran, I. G., Thomas, I. P., \& Norris, D. R. Wild birds learn songs from experimental vocal tutors. Curr. Biol. 28(20), 3273-3278 (2018).

Monier, M., Nöbel, S., Danchin, E., \& Isabel, G. (2019). Dopamine and serotonin are both required for matecopying in Drosophila melanogaster. Frontiers in behavioral neuroscience, 12, 334.

Mooney, R. Neural mechanisms for learned birdsong. Learn. Mem. 16, 655-669 (2009).

Nakatani, Y., Matsumoto, Y., Mori, Y., Hirashima, D., Nishino, H., Arikawa, K., \& Mizunami, M. (2009). Why the carrot is more effective than the stick: different dynamics of punishment memory and reward memory and its possible biological basis. Neurobiology of learning and memory, 92(3), 370-380.

Narula, G., Herbst, J. A., Rychen, J. \& Hahnloser, R. H. R. Learning auditory discriminations from observation is efficient but less robust than learning from experience. Nat. Comm. 9 (2018).

Noudoost, B., \& Moore, T. (2011). The role of neuromodulators in selective attention. Trends in cognitive sciences, 15(12), 585-591.

O’Doherty, J. P. (2004). Reward representations and reward-related learning in the human brain: insights from neuroimaging. Current opinion in neurobiology, 14(6), 769-776.

Orban de Xivry, J. J., Criscimagna-Hemminger, S. E., \& Shadmehr, R. (2011). Contributions of the motor cortex to adaptive control of reaching depend on the perturbation schedule. Cerebral Cortex, 21(7), 14751484.

Panksepp, J. B., Lahvis, G. P. (2007) Social reward among juvenile mice. Genes Brain Behav. 6, 661-671.

Paraouty, N., Charbonneau, J. A., \& Sanes, D. H. (2020). Social learning exploits the available auditory or visual cues. Scientific reports, 10(1), 1-14.

Radhakishun, F. S., van Ree, J. M., \& Westerink, B. H. (1988). Scheduled eating increases dopamine release in the nucleus accumbens of food-deprived rats as assessed with on-line brain dialysis. Neuroscience letters, 85(3), 351-356.

Ripollés, P., Marco-Pallarés, J., Hielscher, U., Mestres-Missé, A., Tempelmann, C., Heinze, H. J., ... \& Noesselt, T. (2014). The role of reward in word learning and its implications for language acquisition. Current Biology, 24(21), 2606-2611.

Ripolles, P., Marco-Pallares, J., Alicart, H., Tempelmann, C., Rodriguez-Fornells, A., \& Noesselt, T. (2016). Intrinsic monitoring of learning success facilitates memory encoding via the activation of the SN/VTAHippocampal loop. Elife, 5, e17441.

Ripollés, P., Ferreri, L., Mas-Herrero, E., Alicart, H., Gómez-Andrés, A., Marco-Pallares, J., ... \& RodriguezFornells, A. (2018). Intrinsically regulated learning is modulated by synaptic dopamine signaling. Elife, 7 , e38113.

Robbins, T. W., \& Everitt, B. J. (2007). A role for mesencephalic dopamine in activation: commentary on Berridge (2006). Psychopharmacology, 191(3), 433-437.

Rodriguiz, R. M., Chu, R., Caron, M. G., Wetsel, W. C. (2004). Aberrant responses in social interaction of dopamine transporter knockout mice. Behav Brain Res 148: 185-198.

Roelfsema, P. R., van Ooyen, A., \& Watanabe, T. (2010). Perceptual learning rules based on reinforcers and attention. Trends in cognitive sciences, 14(2), 64-71.

Roitman, M. F., Stuber, G. D., Phillips, P. E., Wightman, R. M., \& Carelli, R. M. (2004). Dopamine operates as a subsecond modulator of food seeking. Journal of Neuroscience, 24(6), 1265-1271.

Rossato, J. I., Bevilaqua, L. R., Izquierdo, I., Medina, J. H., \& Cammarota, M. (2009). Dopamine controls persistence of long-term memory storage. Science, 325(5943), 1017-1020.

Sasaki, A., Sotnikova, T. D., Gainetdinov, R. R., \& Jarvis, E. D. (2006). Social context-dependent singingregulated dopamine. Journal of Neuroscience, 26(35), 9010-9014. 
585 Sasaki, Y., Nanez, J. E., \& Watanabe, T. (2010). Advances in visual perceptual learning and

586 plasticity. Nature Reviews Neuroscience, 11(1), 53-60.

587 Schultz, W., Dayan, P., \& Montague, P. R. (1997). A neural substrate of prediction and reward. Science, 588 275(5306), 1593-1599.

589 Schultz, W. (2000). Multiple reward signals in the brain. Nature reviews neuroscience, 1(3), 199-207.

590 Schultz, W. (2002). Getting formal with dopamine and reward. Neuron, 36(2), 241-263.

591 Schultz, W. (2006). Behavioral theories and the neurophysiology of reward. Annu. Rev. Psychol., 57, 87-

592115.

593 Schultz, W. (2013). Updating dopamine reward signals. Current opinion in neurobiology, 23(2), 229-238.

594 Schultz, W. (2016). Dopamine reward prediction-error signalling: a two-component response. Nature 595 Reviews Neuroscience, 17(3), 183.

596 Seitz, A., \& Watanabe, T. (2005). A unified model for perceptual learning. Trends in cognitive sciences, 597 9(7), 329-334.

598 Shmuelof, L., Krakauer, J. W., \& Mazzoni, P. (2012). How is a motor skill learned? Change and invariance 599 at the levels of task success and trajectory control. Journal of neurophysiology, 108(2), 578-594.

600 Steinberg, E. E., Keiflin, R., Boivin, J. R., Witten, I. B., Deisseroth, K., \& Janak, P. H. (2013). A causal link 601 between prediction errors, dopamine neurons and learning. Nature neuroscience, 16(7), 966-973.

602 Suzuki, H., \& Lucas, L. R. (2015). Neurochemical correlates of accumbal dopamine D 2 and amygdaloid 5603 HT 1B receptor densities on observational learning of aggression. Cognitive, Affective, \& Behavioral 604 Neuroscience, 15(2), 460-474.

605 Tamir, D. I., \& Hughes, B. L. (2018). Social Rewards: From basic social building blocks to complex social 606 behavior. Perspectives on Psychological Science, 13(6), 700-717.

607 Tempel, B. L., Bonini, N., Dawson, D. R., \& Quinn, W. G. (1983). Reward learning in normal and mutant 608 Drosophila. Proceedings of the National Academy of Sciences, 80(5), 1482-1486.

609 Thiele, A., \& Bellgrove, M. A. (2018). Neuromodulation of attention. Neuron, 97(4), 769-785.

610 Wickens, J. R., Reynolds, J. N., \& Hyland, B. I. (2003). Neural mechanisms of reward-related motor 611 learning. Current opinion in neurobiology, 13(6), 685-690.

612 Yanagihara, S. \& Yazaki-Sugiyama, Y. Social interaction with a tutor modulates responsiveness of specific 613 auditory neurons in juvenile zebra finches. Behav. Processes. 163, 32-36 (2019). 


\section{Figure Legends}

\section{Figure 1}

616 Yoking food reward to an observer facilitates social learning. A. Replication of Figure 1 from Paraouty et 617 al. (2020). Experimental Design. Left: Naïve observer gerbil (black) was separated from a performing samesex demonstrator gerbil (brown) by a transparent divider. The demonstrator was previously trained by the experimenters (see Methods for details). Right: Following five days of exposure, the practice phase began and mean (thick lines) \pm standard error of the mean (SEM) of overall number of Go (dark color) and Nogo trials (pale color) performed by the demonstrators ( 5 males; age $=122.6 \pm 4.7$ days (mean \pm standard error)) during the 5 days of exposure (brown), and by the naïve observer gerbils (age $=101.4 \pm 4.1$ ) during the practice sessions (black). C. Individual (thin lines) and mean \pm SEM (thick lines) performance d' values of the demonstrator during the 5 days of exposure (brown) and of the naïve observer gerbil during the practice sessions (black). The performance sensitivity, d' was calculated once the observers performed $>15$ Nogo trials. D. Experimental Design. Left: Yoked reward observer gerbil (orange) was separated from a performing demonstrator gerbil (brown) by a transparent divider. A food tray was also present in the observer's compartment, and the food reward of the demonstrator animal was yoked to that of the observer.

Right: Practice session, similar to A (right). E. similar to B, with demonstrators (4 males; age $=130 \pm 4.2)$ and yoked reward observers (age $=96.8 \pm 5.3)$. F. similar to $\mathrm{C}$ for the yoked reward observers. The pale grey line indicates the mean d' of the naïve observers without the yoked reward (from Figure 1C).

\section{Figure 2}

Decrease of tonic dopamine level degrades social learning. A. Prior to each exposure session, the naïve observer gerbils were given an injection of a dopamine receptor antagonist ( 5 males; age $=99.5 \pm 8.6$ days; see Methods for details). After 15-20 min of recovery, the exposure session began (left) with same-sex 
640 (left) with the demonstrators (age=143 \pm 5.4 ). No drug was injected during the practice sessions (right). C.

641 The performance of the observer gerbils which received a dopamine antagonist (red) and those that received saline (blue) as a function of practice sessions. D. Prior to each exposure session, the yoked reward observer gerbils were given an injection of a dopamine receptor antagonist (4 males; age=111.3 \pm 8.9 ). After 15-20 min of recovery, the exposure session began (left) with the demonstrators (age $=166 \pm 4.5$ ). No drug was injected during the practice sessions (right). E. Similar to D, except that saline was injected to the observers (5 males; age $=110.3 \pm 8.5$ ) prior to each exposure session (left) with the demonstrators (age $=119 \pm 5.7$ ). No drug was injected during the practice sessions (right). F. The performance of the yoked reward observer gerbils which received a dopamine antagonist (red) and those that received saline (blue) as a function of practice sessions.

Figure 3

Increase of tonic dopamine level enhances social learning. A. Prior to each exposure session, the naïve observer gerbils were given an injection of a dopamine receptor agonist ( 6 males; age $=100.3 \pm 12$ days; see Methods for details). After 15-20 min of recovery, the exposure session began (left) with the demonstrators (age $=139 \pm 5.2$ ). No drug was injected during the practice sessions (right). B. Similar to A, except that saline was injected to the observers ( 5 males; age $=104.3 \pm 10.7)$ prior to each exposure session (left) with the demonstrators (age $=163 \pm 5.4$ ). No drug was injected during the practice sessions (right). C. The performance of the observer gerbils which received a dopamine agonist (green) and those that received saline (blue) as a function of practice sessions.

\section{Figure 4}

Synchrony between vocalization and sound onset may provide key social cues to observers. A. Example of 
666

667

668

669

670

671

672

673

674

675

676

677

678

679

680

681

682

683

684

685

686

687

688

subset of 5 demonstrators in each experimental condition as a function of the onset of the Go and Nogo sound stimuli. The inset represents the percent of demonstrators' calls that were present within $\pm 0.5 \mathrm{~s}$ of the sound onset (dark grey bars). E. Histogram of vocalization onsets for a subset of 5 observers in each experimental condition as a function of the onset of the Go and Nogo sound stimuli (the same demonstratorobserver pairs recordings were analyzed). The inset represents the percent of observers' calls that were present within $\pm 0.5 \mathrm{~s}$ of the sound onset (dark grey bars). F. Mean number of demonstrators' calls within $\pm 0.5 \mathrm{~s}$ of the sound onset plotted against the number of days taken by the respective observers to reach the criterion d' of 1.5. The data is from 5 demonstrator and observer pairs in each of the seven experimental groups tested (average of the five daily exposure sessions). G. Mean number of demonstrators' calls unsynchronized to the sound onset plotted against the number of days taken by the respective observers to reach the criterion d'. Asterisks denote statistically significant post-hoc differences at the following levels: * $p<0.05$ and $* * p<0.01$.

\section{Video legend}

\section{Video 1}

Social learning of an auditory discrimination task. A naïve observer gerbil was permitted to experience a trained conspecific demonstrator perform an auditory Go-Nogo discrimination task. The naïve observer gerbil was exposed to a minimum of 80 Go trials and 20 Nogo trials in each exposure session. The performance of the demonstrator during those 5 exposure sessions is shown in Figure 1 (brown lines). Following five such exposure sessions, the naive observer gerbil was subsequently permitted to practice the task. The performance of the observer gerbil during the subsequent practice sessions is shown in Figure 1 (black lines). The markings on top of the demonstrator and the observer gerbil were obtained using DeepLabCut with 4 labelled features marked: nose tip, left ear, right ear, and tail base. 


\section{Supplementary Information}

690

\section{Supplementary Figure 1}

Administration of dopamine antagonist impacts the general behavior of observers. A. Illustration of the orientation for the $0-18^{\circ}$ which corresponds to the food tray for the same subset of 5 observers (as in B). Asterisks denote statistically significant post-hoc differences at the following levels: ${ }^{*} p<0.05$ and ${ }^{* *} p<0.01$.

\section{Supplementary Figure 2}

D1/D5 antagonist injections during exposure negatively impacts subsequent performance. A. Mean total number of trials $( \pm$ SEM) initiated by all observers in the seven different experimental groups of observers across practice days (Go and Nogo trials). B. Mean hit rate ( \pm SEM) of all observers across practice days. A mixed-model ANOVA revealed significant group differences, and post-hoc comparisons confirmed the significantly poorer Hit rates of the D1/D5 antagonist observer groups as compared to all other groups $(p<0.001)$ during the early days of practice. No significant group difference was found between the other observer groups.

\section{Supplementary Figure 3}


bioRxiv preprint doi: https://doi.org/10.1101/2021.03.03.433719; this version posted March 3, 2021. The copyright holder for this preprint (which was not certified by peer review) is the author/funder. All rights reserved. No reuse allowed without permission.

Figure 1

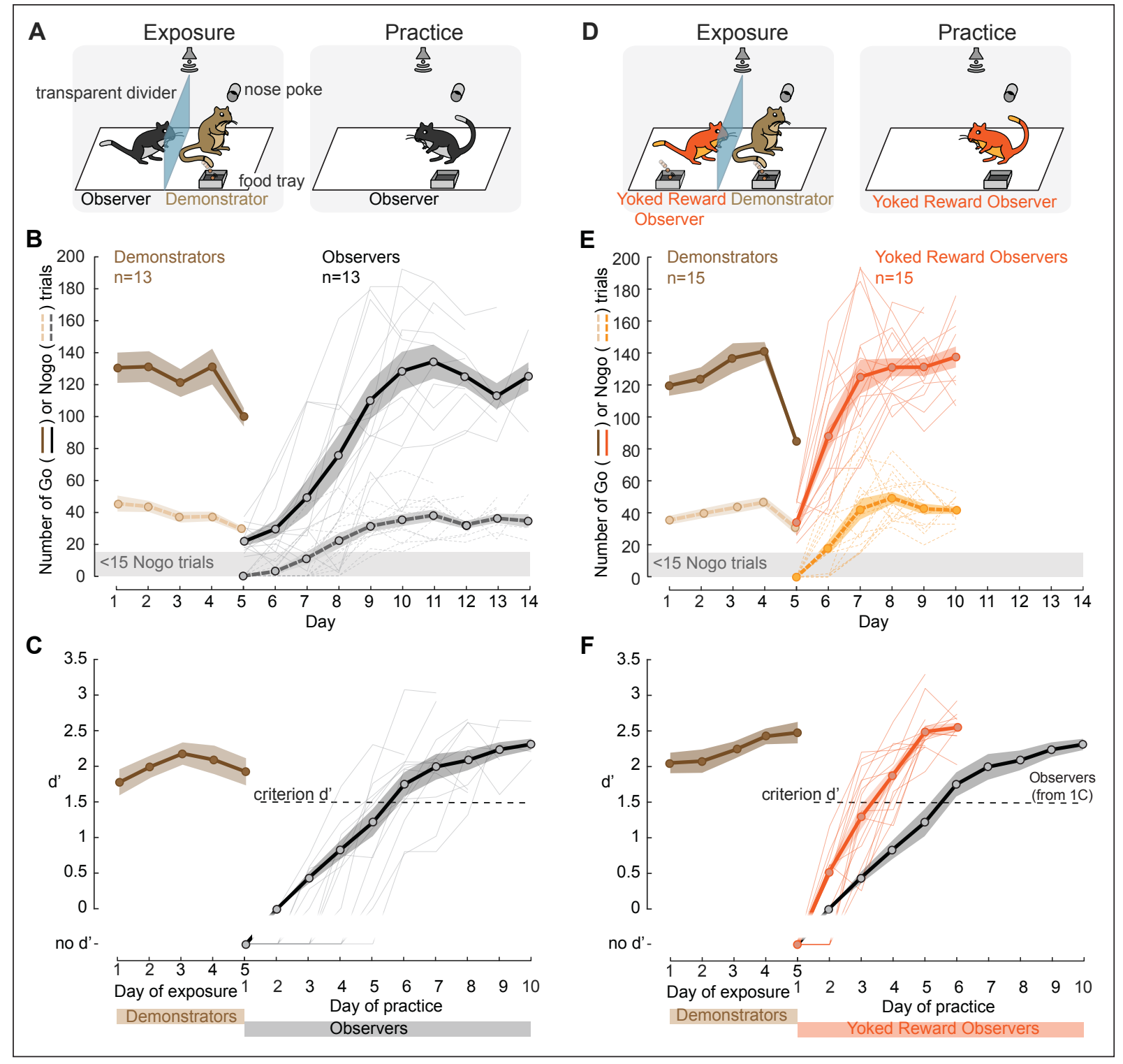


bioRxiv preprint doi: https://doi.org/10.1101/2021.03.03.433719; this version posted March 3, 2021. The copyright holder for this preprint (which was not certified by peer review) is the author/funder. All rights reserved. No reuse allowed without permission.

Figure 2

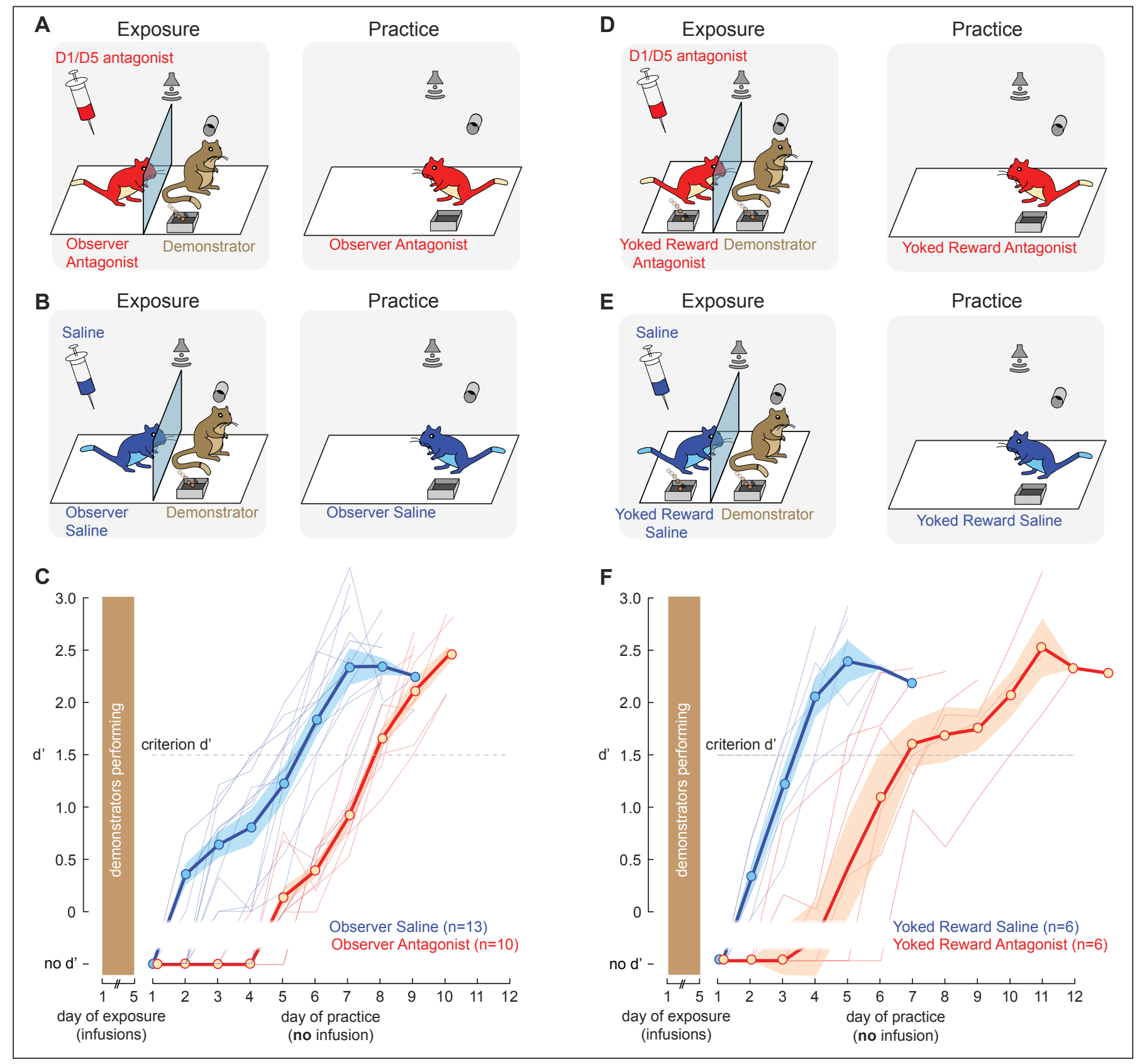


bioRxiv preprint doi: https://doi.org/10.1101/2021.03.03.433719; this version posted March 3, 2021. The copyright holder for this preprint (which was not certified by peer review) is the author/funder. All rights reserved. No reuse allowed without permission.

Figure 3

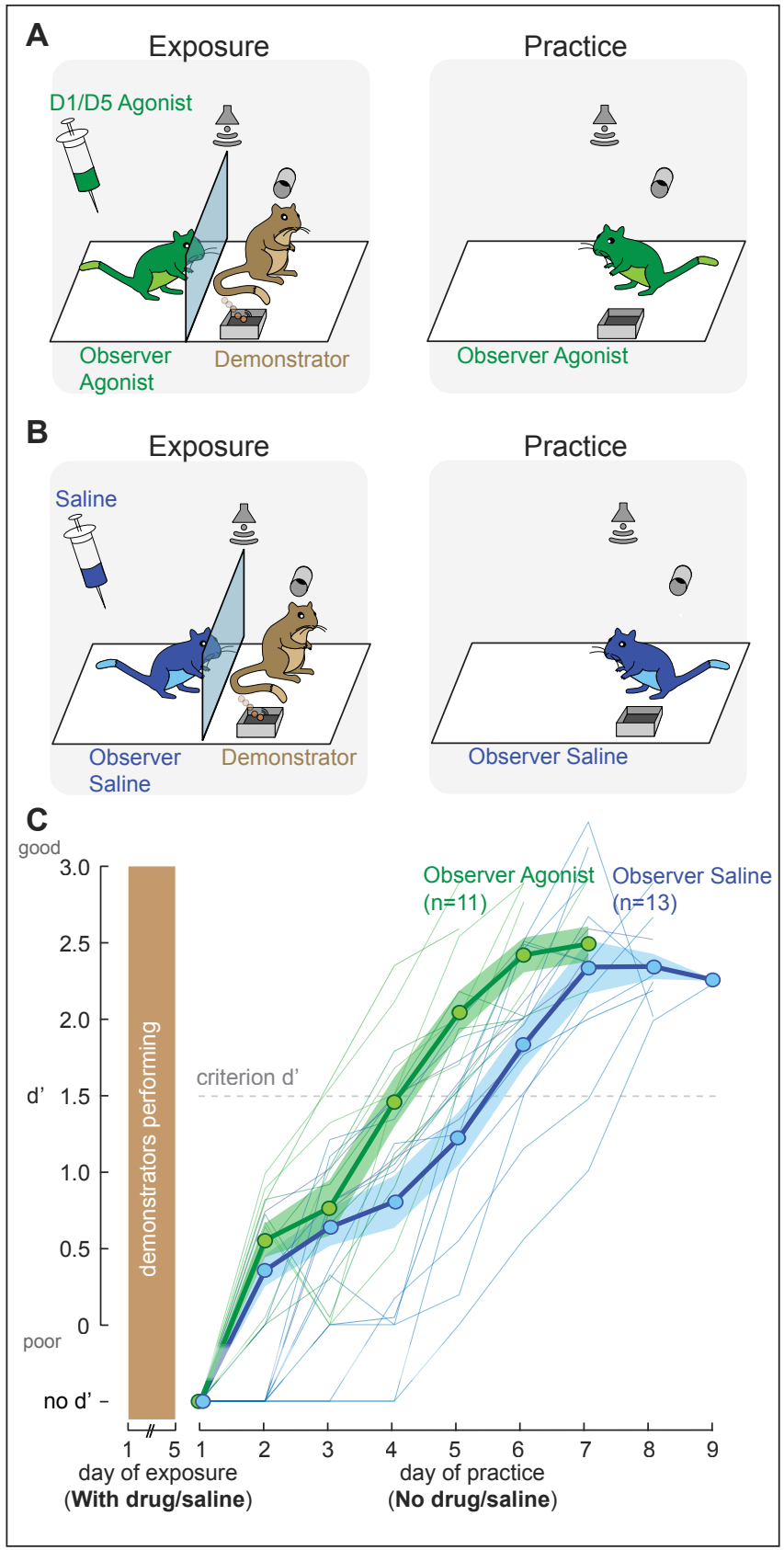


Figure 4

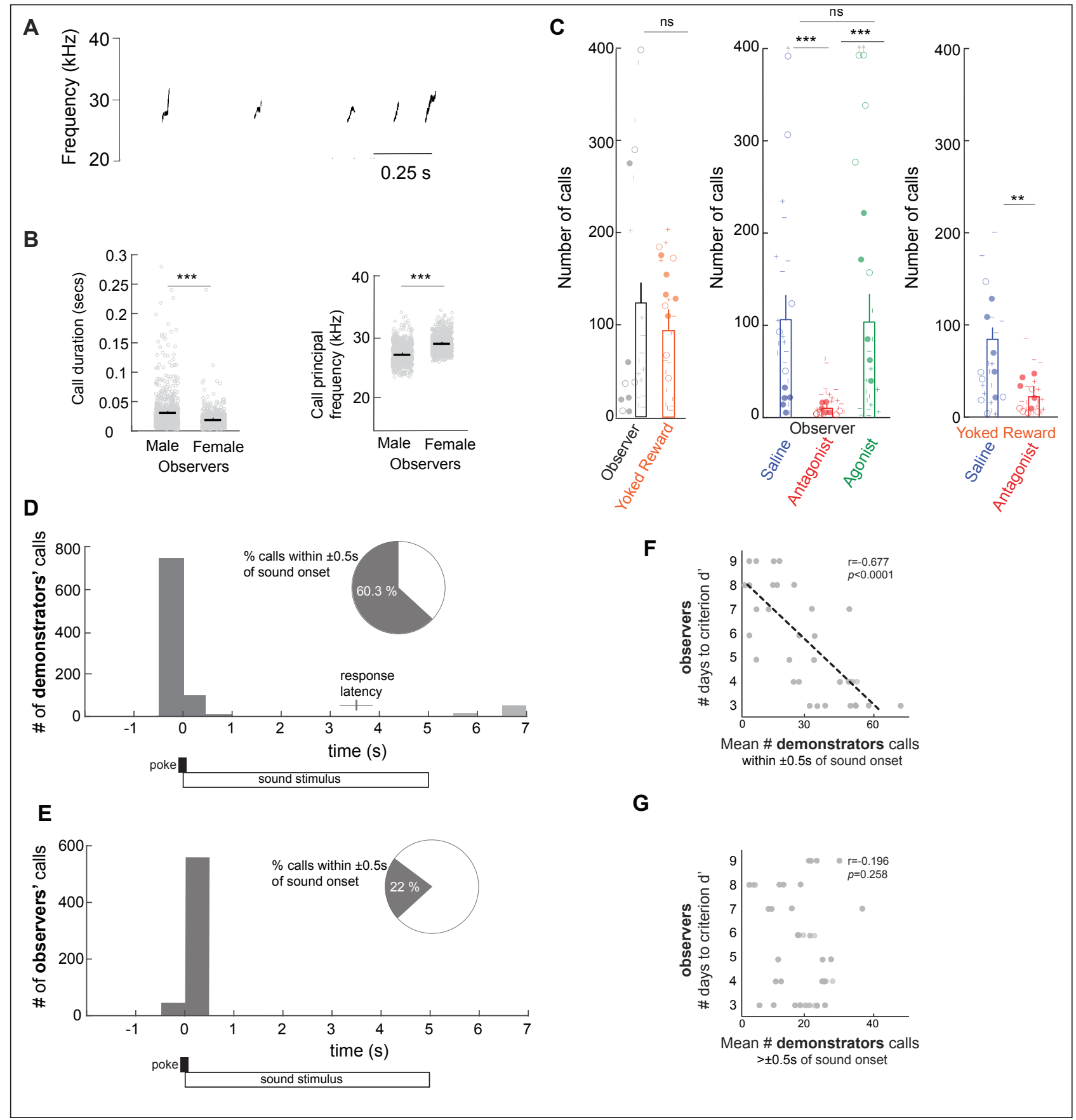

